\title{
Design cost-effective genome-wide and candidate gene association studies of mother-child pairs
}

\author{
Haitao Zheng, Cuilin Zhang, Yun Lu and Jinbo Chen*
}

A case-control mother-child pair design is popular for genetic association studies of obstetric or neonatal outcomes because it allows assessment of both maternal and offspring genotype effects. But a practical constraint is the high genotyping cost, which may double that for a study with the same number of unrelated cases and controls. This issue is particularly relevant for genome-wide association studies, despite the decreasing genotyping cost. Here, we developed cost-effective genotyping strategies for the case-control mother-child pair design. We assumed that the maternal genome had been genotyped a priori, and that the primary goal was to detect marginal offspring genotype effects. Our results indicated that only offspring of case mothers needed to be genotyped in order to achieve a statistical power similar to that for screening the maternal genome. Our conclusion was based on a novel score statistic for testing offspring genotype effects that has a higher power than the Armitage trend test.

KEYWORDS AND PHRASES: Mother-child pairs, Maternal genotype effect, Offspring genotype effect, One-stage design, Two-stage design.

\section{INTRODUCTION}

Obstetric complications and neonatal outcomes are complex traits that may involve multiple genes in their etiology. Because both maternal and fetal genes regulate the intrauterine environment, which in turn plays a critical role in both maternal and fetal health, it has been of interest to assess the contribution of both maternal and offspring genomes to the risk of adverse obstetric and neonatal outcomes (e.g., Weinberg 1998; Wilcox 1998; Saftlas AF, 2005; Takimoto E, 1996; Wangler MF, 2005). Emerging evidence indicated that it has indeed been fruitful to look beyond mothers' or fetuses' own genomes. For instance, it has been shown that fetal genes influence maternal metabolism and blood pressure during pregnancy among women carrying fetuses with Beckwith Wiedemann syndrome (Wangler MF, 2005), and the impact of fetal genes on maternal physiology has also been demonstrated by animal models (Kanayama N, 2002; Petry CJ, 2007; Petry et al., 2010). Recent candidate gene and genomewide association studies (GWAs) have

* Corresponding author. identified susceptible SNPs in both maternal and offspring genomes in relation to multiple phenotypes, such as preterm labor and autism. For example, maternal genes TIMP2 and COL4A3 and fetal genes IL6R, IGF2, IL2, HMGCR, and APOA1 were recently found to be associated with the risk of spontaneous preterm labor (Romero et al., 2010; Steffen et al., 2007). The maternal RFC1 gene was found to be associated with the risk of autism (James et al., 2010), and both maternal and offspring MAOA genes were found to be associated with autism severity (Cohen et al., 2010).

Traditionally, family-based study designs have been adopted for assessing both maternal and offspring genotype effects (OGEs), and standard case-control designs have been adopted to assess only maternal effects or OGEs. However, genetic association studies based on mother-child pairs represent a unique advantage. They allow assessment of both maternal and offspring genes, and yet are cost effective because they do not need to recruit fathers as in family-based studies. However, a practical constraint of such a design is the high genotyping cost, since two genomes need to be genotyped for each subject. The high cost is particularly relevant in GWAs which involve millions of genetic markers, despite the decreasing genotyping cost. Here, we investigate costeffective genotyping designs for GWAs and candidate gene studies involving case-control mother-child pairs.

We focus on genotyping designs for testing OGEs, recognizing that results are readily extensible for testing maternal genotype effects (MGEs). Cases and controls are mothers who did or did not develop the phenotype of interest. DNA samples for their children are available. The standard genotyping design is to genotype all case-control mother-child pairs. However, although both MGEs and OGEs are important, investigators may first examine subjects' own genomes, that is, the maternal genome. In fact, a majority of published genetic association studies of obstetric outcomes only afforded to examine the maternal genome. We thus assume that the maternal genome has been genotyped a priori. Our main idea is to exploit the obvious correlation between maternal and offspring genotypes. As such, maternal genotypes can serve as surrogates for offspring genotypes. We thus propose that only a proportion of offspring need to be genotyped and that genetic information for the untyped offspring can be recovered from the maternal genotype data.

We consider cost-effective genotyping designs for both one-stage and two-stage studies of mother-child pairs. Designing cost-effective two-stage genetic association studies 
has recently been an important research topic (Satagopan et al., 2004; Wang et al., 2006; Skol et al., 2007). These designs examine a panel of SNPs only on a subset of study subjects (stage I), then a small percentage of promising SNPs are genotyped for the rest of the subjects (stage II). With a judiciously selected proportion of stage-I subjects and critical points for testing significance, joint analysis of data from the two stages can have a power similar to the design where all subjects are fully genotyped (Skol et al., 2006). To investigate the influence of the offspring genome, the simplest genotyping design would be to use exactly the same design as that for the maternal genome. That is, children of stage I (II) mothers would be genotyped in stage I (II). However, based on a powerful score statistic we developed, we show here that it is feasible to further reduce the genotyping cost. In particular, we demonstrate that it suffices to genotype children of cases and perhaps a small proportion of children of controls.

\section{MATERIAL AND METHODS}

The selection of a cost-effective design obviously depends on the selection of test statistics. With data from a casecontrol study, it is common to test the association between a SNP and the binary phenotype using the standard Armitage trend statistic (Armitage, 1955). We adopt a score-based statistic to screen the maternal genome for multiplicative genotype effects, which has a power similar to that of the Armitage trend test (Chen and Chatterjee, 2007). For testing marginal multiplicative effects of offspring genotypes, we develop a score-based statistic adapted from a likelihood ratio statistic proposed in our previous work (Chen et al., 2009), which is computationally more efficient and yet has a similar statistical power. This statistic integrates maternal genotype data into the test of OGEs, thereby improving the test power and making it possible to reduce the cost of genotyping offspring. We recognize that testing OGEs on maternal phenotypes may require appropriate adjustment of maternal effects. When a maternal SNP has a significant marginal effect, the corresponding offspring genotype could also be significant since it is correlated with the maternal genotype. We consider two simple approaches to adjusting for MGEs: one is to stratify on the maternal genotype, and the other is to include the maternal effect in the penetrance model. Of course, when neither the maternal nor the offspring SNP is associated with the phenotype, unadjusted analyses would maintain nominal type-I error rates. We compare the power of the two approaches in the setting of our proposed design.

\subsection{Notation}

We consider the testing of a bi-allelic SNP and refer to the common allele as $A$ and minor allele as $a$. The three possible genotypes are $A A(0), A a(1)$, and $a a(2)$. A maternal and offspring SNP at the same locus have seven possible combinations of genotypes: $(0,0),(0,1),(1,0),(1,1)$
$(1,2),(2,1)$, and $(2,2)$. Let $Y$ denote the binary phenotype that takes value " 1 " for cases and "0" for controls. We assume that $n_{1}$ case mother-child pairs and $n_{0}$ control pairs are recruited. Let $G^{m}$ and $G^{c}$ denote the genotype of a maternal and an offspring SNP, respectively, and let $p_{a}$ be the minor allele frequency (MAF) of $G^{m}$ and $G^{c}$ for control mothers and their children. We assume that the penetrance of $G^{m}$ and $G^{c}$ is quantified by a log-linear model $\log p\left(Y=1 \mid G^{m}, G^{c} ; \beta\right)=\beta_{0}+f\left(G^{m}, G^{c} ; \beta_{1}\right)$, where $f\left(G^{m}, G^{c} ; \beta_{1}\right)$ is a pre-specified relative risk function. We use "null model" to refer to the situation where neither $G^{m}$ nor $G^{c}$ is associated with $Y$ so that $f\left(G^{m}, G^{c} ; \beta_{1}\right)$ is a constant. Let $\beta=\left(\beta_{0}, \beta_{1}\right)$. We use the "marginal maternal model" to refer to the model with only a multiplicative MGE,

$$
\log p\left(Y=1 \mid G^{m} ; \beta\right)=\beta_{0}+\beta_{1} G^{m},
$$

where $G^{m}$ takes values 0,1 , or 2 according to the number of minor alleles at the locus. We similarly use the "marginal offspring model" to refer to the model with only a multiplicative OGE,

$$
\log p\left(Y=1 \mid G^{c} ; \beta\right)=\beta_{0}+\beta_{1} G^{c}
$$

\subsection{The score-based test statistic}

Here, we develop a score-based test statistic for OGEs, on which our design, which genotypes only a subset of children, is based. The statistic uses data $\left(Y, G^{m}, G^{c}\right)$ collected from $n_{1}$ case mothers and $n_{0}$ control mothers and data $\left(Y, G^{c}\right)$ from $n_{1}^{c}$ children of case mothers $\left(n_{1}^{c} \leq n_{1}\right)$ and $n_{0}^{c}$ children of control mothers $\left(n_{0}^{c} \leq n_{0}\right)$. Under the penetrance function (2), the score test statistic of the null hypothesis $\beta_{1}=0$ is based on the following likelihood function:

$$
\begin{aligned}
L\left(\beta_{1}, p_{a}\right)= & \prod_{i=1}^{n_{1}^{c}} \frac{e^{\beta_{1} G_{i}^{c}} p\left(G_{i}^{c}, G_{i}^{m} \mid Y_{i}=0\right)}{\sum_{G^{c}} e^{\beta_{1} G^{c}} p\left(G^{c} \mid Y=0\right)} \\
& \times \prod_{j=1}^{n_{0}^{c}} p\left(G_{j}^{c}, G_{j}^{m} \mid Y_{j}=0\right) \\
& \times \prod_{i=n_{1}^{c}+1}^{n_{1}} \frac{\sum_{G^{c}} e^{\beta_{1} G^{c}} p\left(G^{c}, G_{i}^{m} \mid Y_{i}=0\right)}{\sum_{G^{c}} e^{\beta_{1} G^{c}} p\left(G^{c} \mid Y=0\right)} \\
& \times \prod_{j=n_{0}^{c}+1}^{n_{0}} p\left(G_{j}^{m} \mid Y_{j}=0\right),
\end{aligned}
$$

where $p\left(G^{m}, G^{c} \mid Y=0\right)$ is a function of $p_{a}$ under assumptions of HWE and transmission equilibrium in the control population (Table 1, Chen et al., 2009). In our previous work (Chen et al., 2009), we proposed to use the likelihood ratio statistic, $2\left\{\log L\left(\hat{\beta}_{1}, \hat{p}_{a}\right)-\log L\left(\beta_{1}=0, \hat{p}_{a}^{0}\right)\right\}$ for testing $\beta_{1}=0$, where $\hat{p}_{a}$ and $\hat{p}_{a}^{0}$ are the estimate of the MAF $p_{a}$ under the alternative and null hypothesis, respectively. To facilitate the power calculation for our proposed imbedded two-stage design, we propose to test $\beta_{1}=0$ based on score 
function $\partial \log L\left(\beta_{1}=0, \hat{p}_{a}^{0}\right) / \partial \beta_{1}$, which is asymptotically a normal random variable. This score-based statistic is equivalent to the likelihood ratio statistic considered in Chen et al. (2009) but is computationally simpler since it does not require the estimation of $\beta_{1}$. This statistic exploits maternal data $G^{m}$ and is more powerful than the standard Armitage test that ignores $G^{m}$ (Chen et al., 2009). The design of the study for screening the maternal genome, in which we assume that the study of the offspring genome is embedded, is based on the likelihood

$$
\prod_{i=1}^{n_{1}} \frac{e^{\beta_{1} G_{i}^{m}} p\left(G_{i}^{m} \mid Y_{i}=0\right)}{\sum_{G^{m}} e^{\beta_{1} G^{m}} p\left(G^{m} \mid Y=0\right)} \times \prod_{j=1}^{n_{0}} p\left(G_{j}^{m} \mid Y_{j}=0\right) .
$$

The corresponding score statistic asymptotically has a power similar to that of the Armitage trend test.

\subsubsection{Ignoring maternal effects could inflate type-I error rates for testing offspring SNPs}

Tests of marginal MGEs and OGEs are correlated. Thus, it is necessary to adjust for significant maternal effects when testing offspring genotypes. Consider the penetrance function $p\left(Y=1 \mid G^{m}, G^{c}\right)=p\left(Y=1 \mid G^{m}\right)$ where only the maternal genotype $G^{m}$ is associated with the phenotype $Y$, but not offspring genotype $G^{c}$. Testing $G^{c}$ using $G^{m}$ is plausible since they are correlated. Without knowledge of a relationship between $Y$ and $\left(G^{m}, G^{c}\right)$, one may wrongly assume a model $\log p\left(Y=1 \mid G^{c} ; \gamma_{0}, \gamma_{1}\right)=\gamma_{0}+\gamma_{1} G^{c}$ for testing the effect of $G^{c}$, where $G^{c}$ takes the value of minor allele counts. This is a "mis-specified" model because the true relationship between $Y$ and $G^{c}$ is induced as $\sum_{G^{c}} p\left(Y=1 \mid G^{m}\right) p\left(G^{m} \mid G^{c}\right)$. With the incorrect model $p\left(Y=1 \mid G^{c} ; \gamma_{0}, \gamma_{1}\right)$ and data $\left(Y, G^{m}, G^{c}\right)$, we test the null hypothesis $\gamma_{1}=0$ with the score function of $\gamma_{1}$. To illustrate the point, we assume that all children are genotyped and write the joint likelihood function (incorrectly) as

$$
\begin{aligned}
L_{a}\left(\gamma_{1}, p_{a}\right) \approx & \prod_{i=1}^{n_{1}} \frac{e^{\gamma_{1} G_{i}^{c}} p\left(G_{i}^{m}, G_{i}^{c} \mid Y_{i}=0\right)}{\sum_{G^{m}, G^{c}} e^{\gamma_{1} G^{c}} p\left(G^{m}, G^{c} \mid Y=0\right)} \\
& \times \prod_{j=1}^{n_{0}} p\left(G_{j}^{m}, G_{j}^{c} \mid Y_{j}=0\right),
\end{aligned}
$$

from which we can obtain the score equation for $\gamma_{1}$ :

$$
l_{a}\left(\gamma_{1}\right)=\sum_{i=1}^{n_{1}}\left\{G_{i}^{c}-\sum_{G^{c}} G^{c} \frac{e^{\gamma_{1} G^{c}} p\left(G^{c} \mid Y=0\right)}{\sum_{G^{c}} e^{\gamma_{1} G^{c}} p\left(G^{c} \mid Y=0\right)}\right\} .
$$

If $\gamma_{0}+\gamma_{1} G^{c}$ were equal to $\log p\left(Y=1 \mid G^{c}\right)$, the summation in the bracket would be equal to $\mathrm{E}\left(G^{c} \mid Y=1\right)$. Consequently, $l_{a}\left(\gamma_{1}\right)=0$ would be an unbiased estimating equation of $\gamma_{1}$, based on which a valid test of $\gamma_{1}=0$ can be constructed. But this is not true with the penetrance function $p\left(Y=1 \mid G^{m}, G^{c}\right)=p\left(Y=1 \mid G^{m}\right)$. Consequently, the test of $\gamma_{1}$ based on $l_{a}\left(\gamma_{1}\right)$ has an incorrect type-I error rate, indicating that valid tests of marginal offspring SNPs may require appropriate adjustment of maternal effects.

On the other hand, if $G^{m}$ is independent of the phenotype $Y$ given $G^{c}$, one can test associations between $Y$ and $G^{c}$ with data $\left(Y, G^{m}\right)$. This can be seen by noting that the score function for $\gamma_{1}$ can be written as $\sum_{i=1}^{n_{1}}\left\{\mathrm{E}\left(G^{c} \mid G_{i}^{m}, Y_{i}=1\right)-\mathrm{E}\left(G^{c} \mid Y_{i}=1\right)\right\}$. The power of the test is thus largely determined by the correlation between $G^{m}$ and $G^{c}$ in the case population. This test statistic is used in an alternative genotyping design strategy described below.

\subsubsection{Simple approaches to adjusting for maternal effects when testing offspring effects}

We propose two approaches to controlling for MGEs. The first strategy is to stratify on maternal genotype $G^{m}$ when testing $G^{c}$. We treat each value of $G^{m}$ as a stratum and analyze the association between $Y$ and $G^{c}$ within each stratum. The penetrance function assuming a multiplicative effect of $G^{c}$ within each $G^{m}$ stratum can be written as

$$
\begin{aligned}
\log p\left(Y=1 \mid G^{m}, G^{c}\right)= & \beta_{0}+\beta_{1} I_{G^{m}=1}+\beta_{2} I_{G^{m}=2} \\
& +\alpha_{0} I_{G^{m}=0} G^{c}+\alpha_{1} I_{G^{m}=1} G^{c} \\
& +\alpha_{2} I_{G^{m}=2} G^{c},
\end{aligned}
$$

where "I" is the indicator function. The score-based test for no association with $G^{c}\left(\alpha_{0}=\alpha_{1}=\alpha_{2}=0\right)$ has three degrees of freedom. If data is insufficient to fit this fully stratified model, then an alternative approach is to fit a multiplicative effect on both $G^{m}$ and $G^{c}$ :

$$
\log p\left(Y=1 \mid G^{m}, G^{c}\right)=\beta_{0}+\beta_{1} G^{m}+\beta_{2} G^{c} .
$$

One can test $\beta_{2}=0$ for the association of $G^{c}$ with phenotype $Y$. If the above approaches are applied to all SNPs under investigation, then the overall type-I error rate can be maintained. But as shown in simulation studies below, they have diminished power in the absence of maternal effects. In particular, in GWAs where most maternal SNPs would be expected to be null, performing the adjusted analysis on all SNPs could result in unnecessary loss of power for detecting offspring SNP effects.

\subsection{A one-stage imbedded design for examining the offspring genome}

We first consider the genotyping design for testing the null hypothesis that an offspring SNP is not associated with the phenotype, assuming that the maternal genotype data is available for all cases and controls (one-stage design). Because our statistic exploits both maternal and offspring genetic data for testing OGEs, even without genotyping all children, it is still possible that it has a power similar to that for testing a maternal genome. Let $\pi_{\text {case }}$ and $\pi_{\text {control }}$ denote proportions of the genotyped children of case and 
control mothers, respectively. Our proposed one-stage designs for assessing the offspring genome are characterized by $\pi_{\text {case }}$ and $\pi_{\text {control }}$.

\subsection{A two-stage imbedded design for screening the offspring genome}

Here, we assume that a two-stage study for screening the maternal genome has been conducted a priori using available design strategies (Satagopan et al., 2004; Wang et al., 2006; Skol et al., 2007). We consider cost effective designs for screening the offspring genome that are imbedded in the two-stage design of the maternal genome. Similar to the onestage design above, this imbedded design has a reduced cost for genotyping the offspring genome by incorporating the available maternal genotype data. Building on our one-stage imbedded design, we propose to genotype only a subset of children of case and control mothers in stage I. Promising SNPs identified in stage I are then further evaluated in the remaining children (for whom the maternal genotype data is usually not available), as in the standard two-stage design.

In the standard two-stage design for screening the maternal genome with $n$ case mothers and $n$ control mothers, we test $m$ independent markers at a family-wise error rate 0.05 . In GWAs, $m$ could be $500 \mathrm{k}$, for example. Then $n \pi_{1}$ cases and the same number of controls are genotyped in stage I, and $m \pi_{m}$ top-ranked markers, selected in stage I based on the score test described in Section 2.2, are genotyped for the remaining $2 n\left(1-\pi_{1}\right)$ case and control mothers in stage II. Let $S_{I}$ and $S_{J}$ be the score-based statistic based on stage-I maternal data and on the maternal data combined from both stages, respectively. A maternal SNP is claimed to be associated with the outcome $Y$ if $\left|S_{I}\right|>c_{I}$ and $\left|S_{J}\right|>c_{J}$, where $c_{I}$ and $c_{J}$ are critical values chosen so that $p\left(\left|S_{I}\right|>c_{I}\right)=\pi_{m}$ and $p\left(\left|S_{I}\right|>c_{I},\left|S_{J}\right|>c_{J}\right)=\alpha$ under the null (Skol et al., 2007). To achieve a desired power for the joint analysis, the parameters $\pi_{1}$ and $\pi_{m}$ are determined by the effect size and MAF of the risk SNP, the relative per-SNP genotyping cost in the two stages, etc.

To derive the imbedded two-stage design for assessing the offspring genome, we assume that the per-SNP genotyping cost is the same for the maternal and offspring genomes. In stage I, among $n \pi_{1}$ children of stage I case mothers, only a proportion $\pi_{11}$ are genotyped. Among $n \pi_{1}$ children of stage I control mothers, only a proportion $\pi_{10}$ are genotyped. Thus, the total number of children genotyped in stage I is $n \pi_{1}\left(\pi_{11}+\pi_{10}\right)$. In stage II, $m \pi_{m}$ markers are genotyped in the remaining children (stage II children), the total number of whom is equal to $n\left\{2-\pi_{1}\left(\pi_{11}+\pi_{10}\right)\right\}$. The parameters $\pi_{11}$ and $\pi_{10}$ affect the study power. Here we choose to select $m \pi_{m}$ top-ranked markers from stage I, the same number as that in the study of the maternal genome. Using our scorebased statistic and performing a joint analysis of data from stages I and II, we study the optimal choices of $\pi_{10}$ and $\pi_{11}$ to achieve a desired study power. The details for calculating the power in the joint analysis are provided in Appendix A, where we address the issue that the test statistics used in stages I and II are different since stage I analysis incorporates maternal genotype data.

To evaluate top-ranked offspring SNPs in stage I and II samples, we argue that the top-ranked maternal and offspring SNPs may overlap only by a very small proportion. Let $S_{m}$ and $S_{c}$ be the score statistics for testing MGEs and OGEs using stage-I data, respectively. Assuming that none of the maternal or offspring SNPs are associated, the probability $p\left\{\left|S_{m}\right|>z_{1-\pi_{m} / 2},\left|S_{c}\right|>z_{1-\pi_{m} / 2}\right\}$ is between $\pi_{m}^{2}$ and $\pi_{m}$, where $z_{1-\pi_{m} / 2}$ is the $1-\pi_{m} / 2$ quantile of the standard normal variable. Since $\pi_{m}$ is very small, the probability that more than 10 SNPs, say, are ranked top among both maternal and offspring markers in stage I would also be very small. In other words, we expect that a small number of SNPs would be evaluated in stage II in both maternal and offspring genomes. Therefore, we assume that no maternal genotype data is available for testing OGEs in stage II.

\subsubsection{Two alternative design strategies}

We compare the power of our embedded design with two other intuitive design strategies. One is to first screen the maternal genome using a standard two-stage design, and then only the offspring SNPs at the same loci as the significant maternal SNPs are evaluated to search for significant offspring effects. This is effectively a three-stage design for screening offspring SNPs. The second strategy is to first test each offspring SNP using only stage-I maternal genotype data (see Section 2.2.1 for the test statistic), and then SNPs selected from this first-stage analysis are reassessed using genotype data from all mothers and their children. To calculate the power of the first strategy, let $S_{m I}$ and $S_{m J}$ be the respective score statistics of maternal tests using data from stage I and both stages. Let $S_{c}$ be the score statistic of the OGE, which used maternal and offspring genotype data for all cases and controls. Let $S_{m}$ be the score statistic of the MGE based only on the maternal data for all cases and controls. The power of this three-stage design was calculated as $p\left(\left|S_{m I}\right|>c_{I},\left|S_{m J}\right|>c_{J},\left|S_{c}\right|>c_{3}\right)=p\left(\left|S_{c}\right|>\right.$ $\left.c_{3}||\left|S_{m I}\right|>c_{I},\left|S_{m J}\right|>c_{J}\right) p\left(\left|S_{m I}\right|>c_{I},\left|S_{m J}\right|>c_{J}\right)$, where the conditional probability was approximated as the conditional probability $p\left(\left|S_{c}\right|>c_{3}||\left|S_{m}\right|>c_{m}\right)$. The critical value $c_{3}$ can be determined in a straightforward manner. The critical value $c_{m}$ is determined as $z_{\left(1-10^{-7} / 2\right)}=5.33$, assuming that 500k maternal SNPs are evaluated.

\section{DESIGN OF SIMULATION STUDIES}

We performed extensive simulation studies to evaluate the type-I error rate and power of our imbedded one-stage and two-stage designs in the presence or absence of maternal effects. All tests were conducted at a type-I error rate $10^{-7}$ under the marginal model (2) with $\beta_{0}=-3.5$ and $\beta_{1}=0.43$, unless otherwise specified. We used 1,000 cases and 1,000 controls in all simulations, and the MAF of the 
risk SNP was 0.25. The simulation was repeated 5,000 times for assessing type-I error rates and 1,000 for assessing power. We examined the type-I error rates for testing OGEs in the presence of maternal effects, considering a simple scenario where the true penetrance function was the marginal maternal model (1), but the effect of offspring genotype $G^{c}$ was tested assuming the (incorrect) model (2). In addition, we assessed the power for testing OGEs using only maternal genotype data in the absence of maternal effects at type-I error rates of $10^{-7}, 10^{-4}$, and 0.05 , where the true penetrance model was assumed to be the marginal offspring model (2). Assuming penetrance model (2), we also compared the power of the two proposed approaches to adjusting for maternal effects.

\section{RESULTS}

\subsection{Cost-effective one-stage designs for testing offspring genotypes}

Figure 1 displays the power for testing OGEs as a function of proportions of genotyped case and control children $\left(\pi_{\text {case }}\right.$ and $\left.\pi_{\text {control }}\right)$. Not surprisingly, the power would increase with both increasing $\pi_{\text {case }}$ and $\pi_{\text {control }}$. Interestingly, when we increased $\pi_{\text {case }}$ at a fixed value of $\pi_{\text {control }}$, the power improvement was much more dramatic compared to that with the same increase in $\pi_{\text {control }}$ at the same fixed value of $\pi_{\text {case }}$. For example, with $\pi_{\text {control }}=80 \%$, increasing $\pi_{\text {case }}$ from $20 \%$ to $40 \%$ resulted in $24 \%$ power improvement. But with $\pi_{\text {case }}=80 \%$, increasing $\pi_{\text {control }}$ from $20 \%$ to $40 \%$ resulted in less than $5 \%$ power improvement. Thus, our results indicate that it is desirable to genotype as many case children as possible. To achieve $80 \%$ power for testing a multiplicative OGE in this simulation setup, multiple design strategies can be considered as suggested in Figure 1, but it appeared to be most cost effective to genotype $87 \%$ case children without genotyping any control children. This would lead to a greater than $50 \%$ reduction in the cost of genotyping offspring.

Table 1 presents the type-I error rate and power of different design options identified from Figure 1 for testing offspring risk SNPs with different MAFs. For comparison, we also included the power using the Armitage trend test without incorporating the maternal data, assuming that all children were genotyped (the row "Standard"). The row with $\pi_{\text {case }}=\pi_{\text {control }}=1$ is the power calculated using our score statistic with all case-control mother-child pairs genotyped. We observed that the type-I error rate was close to the nominal level 0.05 in all scenarios. Both cost effective designs we considered, which were defined by $\left(\pi_{\text {case }}, \pi_{\text {control }}\right)=$ $(0.87,0)$ and $\left(\pi_{\text {case }}, \pi_{\text {control }}\right)=(0.80,0.50)$, had a power similar to the "Standard" analysis irrespective of the MAF of the risk allele. Of course, genotyping all children would lead to around a $15 \%$ power increase compared to the two cost-effective designs, but at the expense of more than $50 \%$ of increase in the genotyping cost for the children.

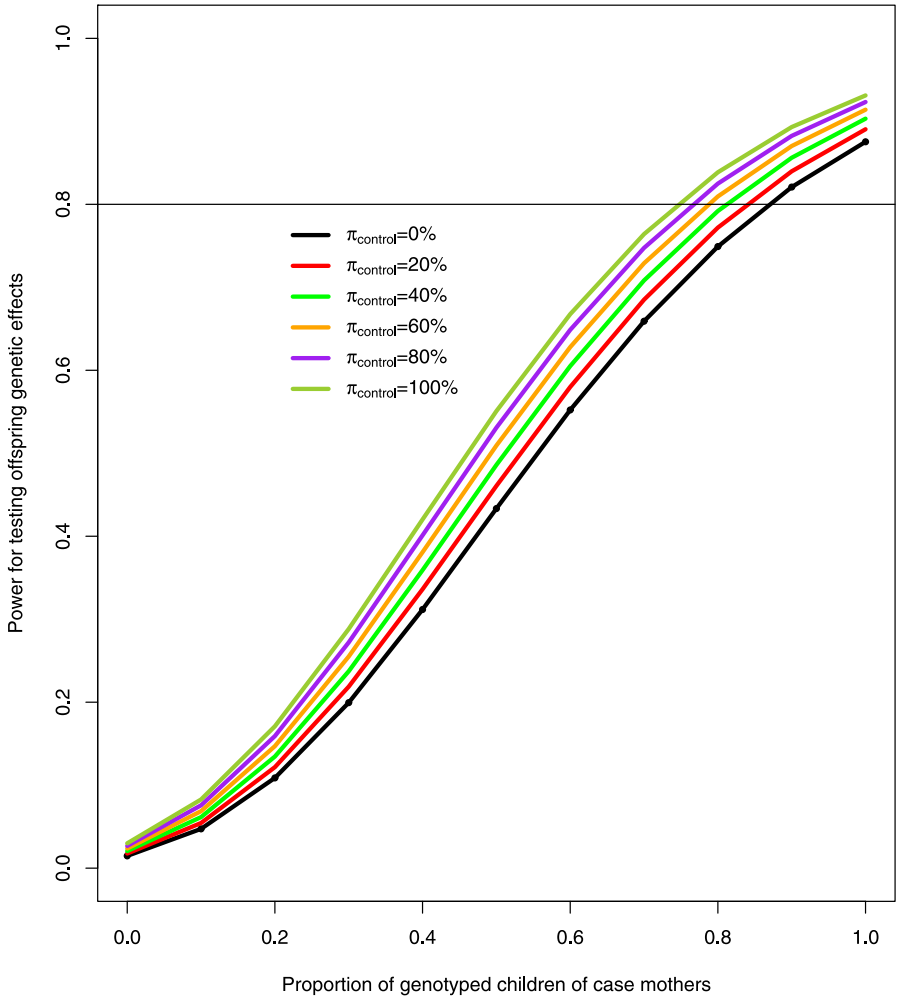

Figure 1. The power of one-stage imbedded design for testing marginal multiplicative offspring genotype effect. The penetrance model was

$\log p\left(Y=1 \mid G^{m}, G^{c}\right)=-3.5+0.43 G^{c}$, the risk SNP MAF was 0.25 , and 1,000 case mother-child pairs and 1,000 control mother-child pairs were used. The test was performed at the significance level $10^{-7}$. Each color line corresponds to a different proportion $\left(\pi_{\text {control }}\right)$ of genotyped children of control mothers.

Table 2 presents the number of case mother-child pairs (assuming an equal number of control mother-child pairs) required to achieve a power $80 \%$ at different cost-effective design options and MAFs of the risk SNP. We also considered sample size requirements with complete genotyping, and with or without exploiting maternal genotype data (the rows corresponding to " $\pi_{\text {case }}=\pi_{\text {control }}=1$ " and "Standard"). Similar to Table 1, it requires a similar number of subjects for the "Standard" method with full genotyping and for the two cost-effective designs. For example, to test a maternal SNP with MAF 0.20 and multiplicative odds ratio 1.54 at $80 \%$ power, 1,148 case pairs are required. To test the corresponding offspring SNP with the same effect size, one would only need to genotype $87 \%$ of case children.

\subsection{Type-I error rates and power for testing OGEs in the presence or absence of MGEs assuming a one-stage design}

Figure 2 presents type-I error rates for testing OGEs. The phenotype data was generated from the marginal maternal 
Table 1. Type-l error rate ${ }^{a}$ and power for testing offspring genotype effects ${ }^{b}$

\begin{tabular}{|c|c|c|c|c|c|c|c|c|c|}
\hline \multirow[b]{2}{*}{$\beta_{1}^{c}$} & \multirow[b]{2}{*}{$\pi_{\text {case }}$} & \multirow[b]{2}{*}{$\pi_{\text {control }}$} & \multicolumn{7}{|c|}{ Minor allele frequency of the risk SNP } \\
\hline & & & 0.05 & 0.10 & 0.15 & 0.20 & 0.25 & 0.35 & 0.50 \\
\hline \multirow{3}{*}{$0^{a}$} & 1.00 & 1.00 & 0.043 & 0.045 & 0.051 & 0.049 & 0.053 & 0.053 & 0.052 \\
\hline & 0.87 & 0.00 & 0.057 & 0.053 & 0.054 & 0.050 & 0.048 & 0.052 & 0.051 \\
\hline & 0.80 & 0.50 & 0.055 & 0.051 & 0.049 & 0.053 & 0.047 & 0.053 & 0.052 \\
\hline & \multicolumn{2}{|c|}{ Standard $^{d}$} & 0.018 & 0.177 & 0.440 & 0.657 & 0.791 & 0.901 & 0.920 \\
\hline \multirow{3}{*}{$0.43^{b}$} & 1.00 & 1.00 & 0.043 & 0.335 & 0.666 & 0.851 & 0.931 & 0.978 & 0.986 \\
\hline & 0.87 & 0.00 & 0.020 & 0.190 & 0.459 & 0.673 & 0.801 & 0.906 & 0.925 \\
\hline & 0.80 & 0.50 & 0.020 & 0.187 & 0.455 & 0.671 & 0.801 & 0.908 & 0.930 \\
\hline
\end{tabular}

${ }^{a}$ The type-I error rate were calculated at the nominal level 0.05 using the same setting as that for Figure 1.

${ }^{b}$ The power calculations were performed using the same setting as that for Figure 1.

${ }^{c}$ The log odds ratio of the multiplicative offspring genotype effect.

${ }^{d}$ Power was calculated assuming that all children were genotyped but maternal genotype data was not used in the test statistic.

Table 2. Number of case mother-child pairs required for testing offspring genotype effects ${ }^{a}$

\begin{tabular}{lcllllll}
\hline \hline & & \multicolumn{5}{c}{ Minor allele frequency of the risk SNP } \\
\cline { 3 - 8 }$\pi_{\text {case }}$ & $\pi_{\text {control }}$ & 0.05 & 0.1 & 0.15 & 0.2 & 0.25 & 0.35 \\
1.00 & Standard $^{b}$ & 3640 & 1961 & 1412 & 1148 & 1000 & 858 \\
0.87 & 1.00 & 2920 & 1590 & 1150 & 940 & 820 & 710 \\
0.80 & 0.00 & 3540 & 1925 & 1396 & 1142 & 1000 & 863 \\
\hline
\end{tabular}

${ }^{a}$ The calculations were performed exactly in the same setting as that for Figure 1.

${ }^{b}$ Power was calculated assuming that all children were genotyped but maternal genotype data was not used in the test statistic.

penetrance model $\log p\left(Y=1 \mid G^{m}, G^{c}\right)=-3.5+0.43 G^{m}$, but in the analysis of offspring genotypes, we assumed a mis-specified penetrance model $\log p\left(Y=1 \mid G^{m}, G^{c}\right)=$ $\gamma_{0}+\gamma_{1} G^{c}$. The three panels from the left to right in Figure 2 display results corresponding to significance levels $10^{-7}$, $10^{-4}$, and 0.05 , respectively. It appeared that the smaller the proportion of genotyped case children was, the more serious the inflation in the type-I error rate for testing the OGE was. For example, at the significance level $10^{-7}$, if only $20 \%$ of case children were genotyped, the empirical type-I error rate was $0.07 \sim 0.20$ regardless of the proportion of genotyped control children. The inflation was serious even when the proportion of genotyped case offspring was close to 1. For example, the type-I error rate was about 0.002 when all mother-child pairs were genotyped, but 0.002 is much greater than the nominal significance level $10^{-7}$.

Figure 3 presents power for testing OGEs when the maternal effect is adjusted for using the two proposed approaches assuming a one-stage design. Here, we used exactly the same parameter setting as that for Figure 1, where no maternal effect was present. In the left panel, the test was stratified on the maternal genotype. In the right panel, a multiplicative maternal effect was adjusted for in the penetrance model. Both approaches appeared to maintain correct type-I error rates, and we omit results here. The first approach appeared to have lower power due to greater degrees of freedom, and the power loss could be as high as $20 \%$ compared with the unadjusted analysis. For the design corresponding to $\pi_{\text {case }}=0.87$ and $\pi_{\text {control }}=0$, compared with the unadjusted analysis, the power loss for the first approach was about $20 \%$, and that for the second approach was about $4 \%$.

\subsection{Power of imbedded two-stage designs for testing OGEs}

Figure 4 presents the power of our proposed imbedded two-stage design at different proportions of genotyped case and control children in stage I. We used the same simulation setup as that for Figure 1. In addition, we chose the proportion of subjects included in stage I to be $\pi_{1}=36.63 \%$ and the proportion of markers genotyped in stage II to be $\pi_{m}=0.83 \%$. The ratio of per-SNP genotyping cost between stages II and I was chosen to be 10. Assuming that none of the 500k maternal SNPs were associated with the phenotype, we assessed the power for identifying an offspring risk SNP with the MAF 0.25 and penetrance function $\log p\left(Y=1 \mid G^{m}, G^{c}\right)=-3.5+0.43 G^{c}$. For our imbedded design, the critical points for calculating power in stages I and II $\left(c_{I}\right.$ and $\left.c_{J}\right)$ were 2.641 and 5.285 , respectively. These values were the same as those for testing the maternal genome, at which the joint analysis of stages I and II data has $80 \%$ power. Similar to the one-stage design, our results suggest that one only needs to genotype about $87 \%$ of case children in stage I in order to achieve $80 \%$ power for identifying the risk offspring SNP. Table 3 presents numbers of case 

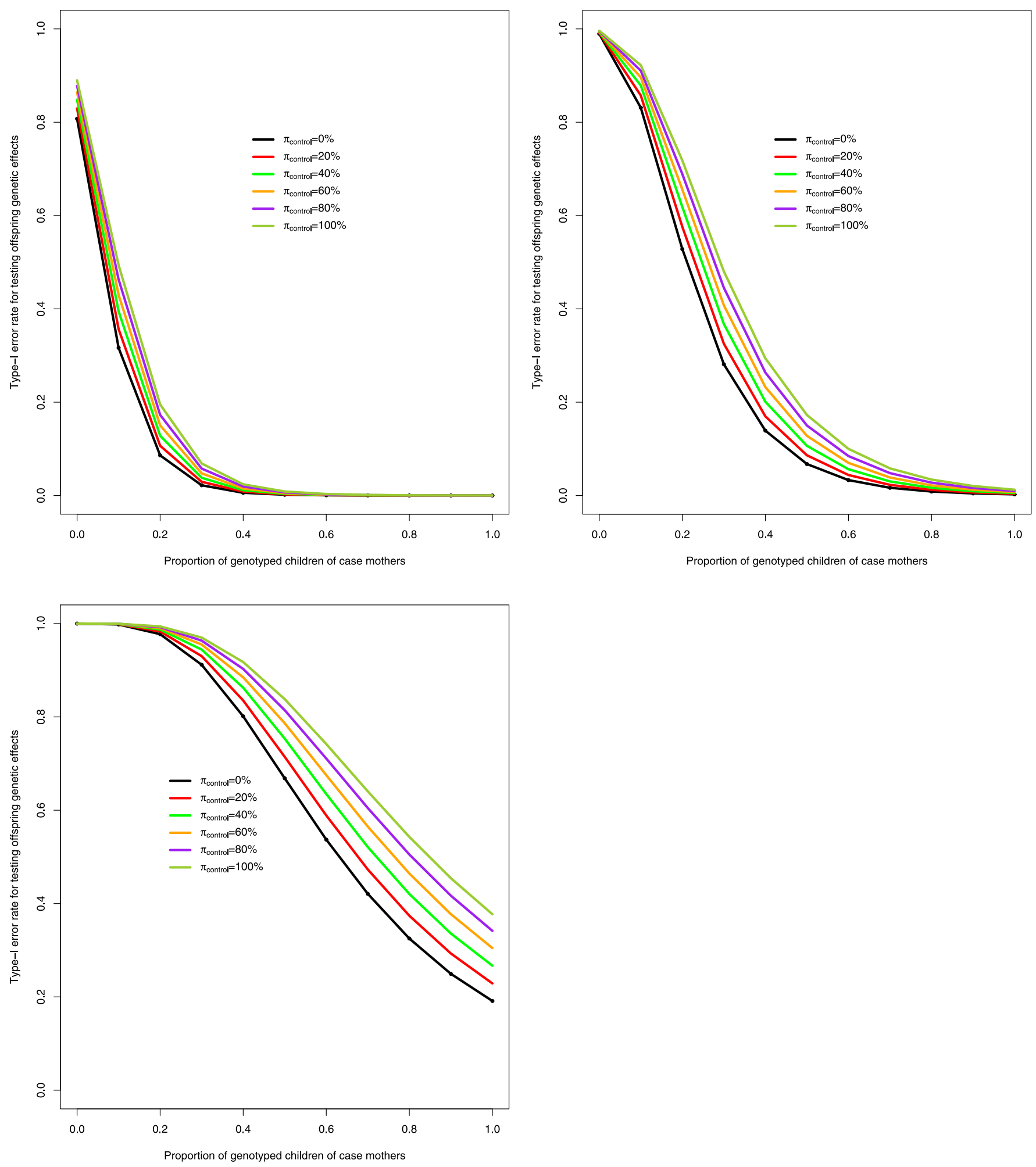

Figure 2. Empirical type-I error rates for testing marginal multiplicative offspring genotype effect assuming a one-stage design. The true penetrance model was the marginal multiplicative maternal model $\log p\left(Y=1 \mid G^{m}, G^{c}\right)=-3.5+0.43 G^{m}$, and the MAF of $G^{m}$ was 0.25 . We used 1,000 case mother-child pairs and 1,000 control mother-child pairs. Each color line in all three panels corresponds to a different proportion of genotyped children of control mothers, and the three panels from left to right corresponds to significance levels $10^{-7}, 10^{-4}$, and 0.05 . The empirical type-l error rate was obtained using simulation that was repeated 5000 times. 

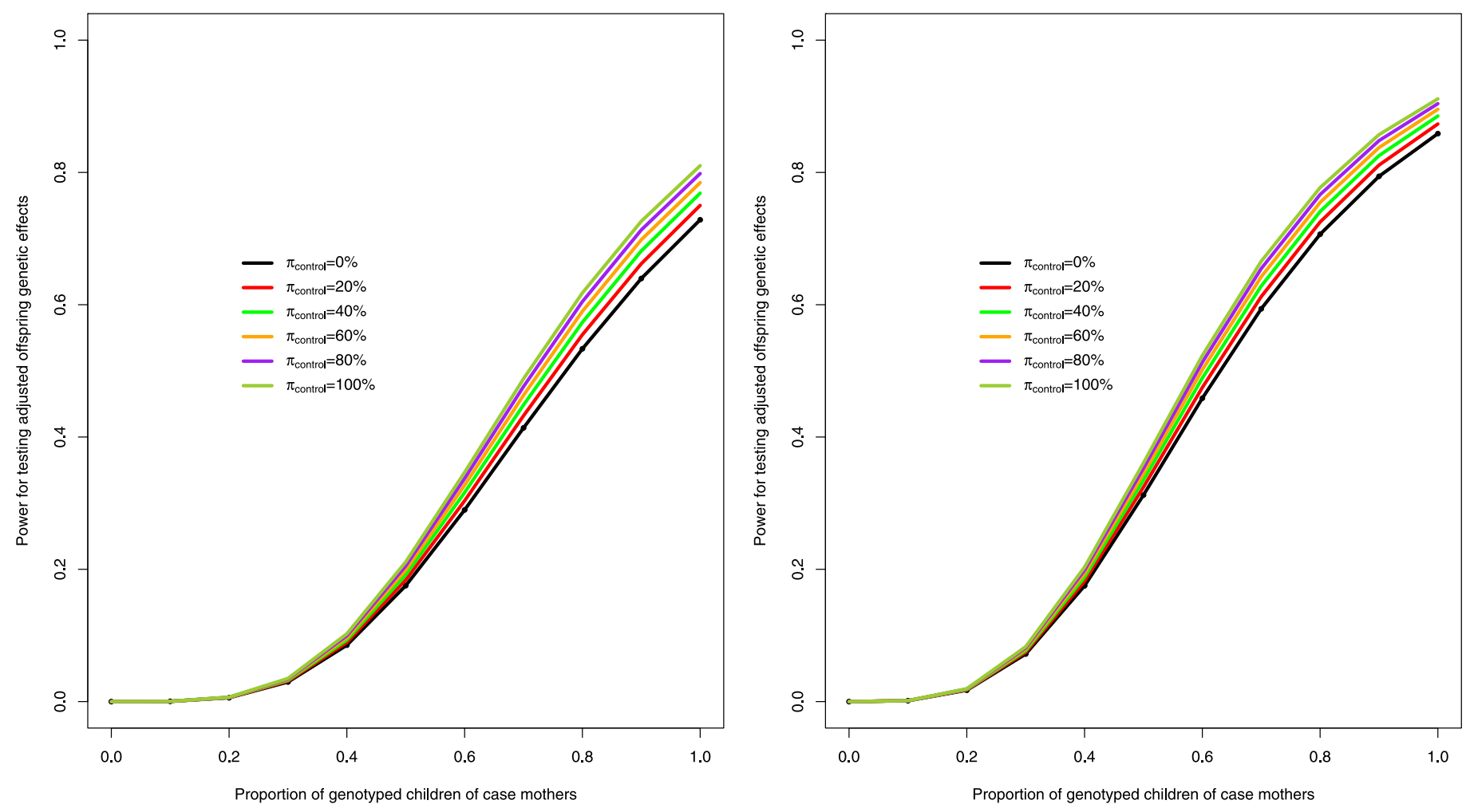

Figure 3. Power for testing offspring genotype effects after adjusting for maternal effects. The true penetrance model was $\log p\left(Y=1 \mid G^{m}, G^{c}\right)=-3.5+0.43 G^{c}$, and the MAF was 0.25 .1000 case mother-child pairs and 1000 control mother-child pairs were used. The left and right panels correspond to the test stratified on maternal genotypes and that adjusting for a multiplicative maternal effect. Each color line corresponds to a different proportion of genotyped children of control mothers. The test was performed at significance level $10^{-7}$.

mother-child pairs required to achieve power $80 \%$ under the imbedded design with $\pi_{11}=0.87$ and $\pi_{10}=0$. In the same scenario, the power of the two alternative two-stage design strategies (see the methods section) were 0.22 and 0.02 , respectively.

\section{DISCUSSION}

In the present study, we investigated cost-effective genotyping designs for GWAs and candidate-gene studies involving mother-child pairs. The designs we proposed for studying the offspring genome are nested within a case-control study where the maternal genotype data is available. Our design could achieve a power similar to that for examining the maternal genome but at a significantly reduced genotyping cost. This reduction owes to the fact that our test statistic incorporates available maternal genotype data for testing OGEs and consequently has an improved power. If a one-stage design is adopted for assessing the maternal genome, then our cost-effective design for genotyping the offspring could save more than $50 \%$ of the genotyping cost compared to the design that genotypes all offspring. In a two-stage design where the ratio of per-SNP genotyping cost between stages II and I is 10, the imbedded two-stage design for the offspring genome could reduce the cost by $\left\{\pi_{1}+10 \pi_{m}\left(1-\pi_{1}\right)\right\}^{-1}\left\{\pi_{1}\left(\pi_{11}+\pi_{10}\right) / 2+10 \pi_{m}\left(1-\pi_{1}\right)\right\}$. For example, with $\pi_{11}=0.87, \pi_{10}=0, \pi_{1}=0.366$, and $\pi_{m}=0.00828$, the saving is close to $50 \%$. In practice, one can genotype all children of case mothers in a one-stage design or all children of stage-I case mothers in a two-stage design. This simple design could maintain a power similar to that for screening the maternal genome but at more than $40 \%$ reduction in the genotyping cost. The same design can naturally be generalized to the study of neonatal outcomes, where a study of the maternal genome could be nested within that of the offspring genome.

It has long been recognized that MGEs and OGEs could confound each other. We studied the inflation in type-I error rates for testing OGEs in the setting of our proposed imbedded cost effective design. We observed an interesting phenomenon that the inflation became more serious as the proportion of genotyped children of case mothers decreased. We found that the analysis that adjusted for a multiplicative maternal effect in the penetrance model appeared to have a power similar to that of the unadjusted analysis in the absence of maternal effects. In general, we recommend that MGEs be ignored when designing a study. For data analy- 
Table 3. Number of case mother-child pairs required in the imbedded two-stage design for screening the offspring genome $\left(\pi_{11}=0.87, \pi_{10}=0\right)$

\begin{tabular}{llllll}
\hline \hline & \multicolumn{5}{c}{ Minor allele frequency of the risk SNP } \\
\cline { 2 - 6 } Odds ratio & 0.05 & 0.15 & 0.25 & 0.35 & 0.50 \\
\hline 1.20 & $-{ }^{a}$ & 9000 & 6000 & 5000 & 4800 \\
1.50 & 4200 & 1600 & 1200 & 1000 & 1000 \\
1.80 & 1900 & 750 & 550 & 480 & 480 \\
2.00 & 1300 & 500 & 400 & 350 & 350 \\
\hline
\end{tabular}

${ }^{a}$ The required number was unrealistically large.

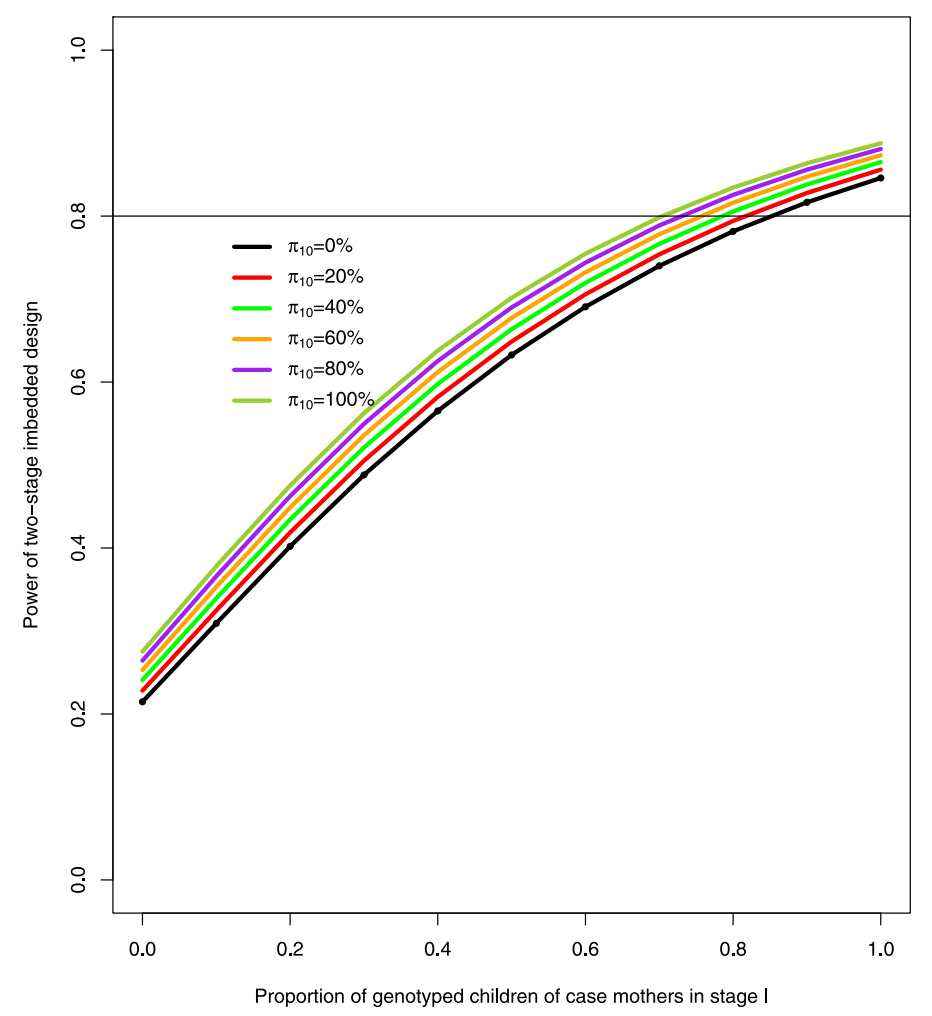

Figure 4. Power of the two-stage imbedded design for screening the offspring genome. The penetrance model was $\log p\left(Y=1 \mid G^{m}, G^{c}\right)=-3.5+0.43 G^{c}$, and the MAF of the risk SNP was 0.25 . 1000 case mother-child pairs and 1000 control mother-child pairs were used. It was assumed that $36.63 \%$ of case and control mothers were genotyped in stage I, and $0.83 \%$ of SNPs were genotyped in stage-II mothers and children. Each color line corresponds to a different proportion of genotyped children of stage-I control mothers. The test was performed at the significance level $10^{-7}$.

sis, an unadjusted analysis could be performed first. Then only significant results would be re-examined with adjusted analysis to avoid unnecessary loss of statistical power.

Our design was proposed for assessing marginal OGEs. We did not extensively examine the power of our design for detecting maternal-offspring gene-gene interactions. But we observed in limited simulation studies that genotyping only children of case mothers could lead to unsatisfactory power for detecting maternal-offspring gene-gene interactions. The design that genotypes a proportion of children of control mothers usually has a higher power. Future work is warranted for developing cost effective designs for detecting mother-offspring gene-gene interactions.

\section{APPENDIX A. POWER CALCULATION FOR ONE-STAGE AND TWO-STAGE DESIGNS UNDER A MARGINAL OFFSPRING MODEL}

We here focus on the method for power calculation under the two-stage design, as is readily seen below, that under the one-stage design is a special case. We assume an equal number of case and control pairs, $n$, to simplify the notation. Following notations in the text, a proportion of $\pi_{11}\left(\pi_{10}\right)$ children are genotyped for $n \pi_{1}\left(n \pi_{1}\right)$ stage-I case (control) mothers. The number of available stage II children is $n\left\{2-\pi_{1}\left(\pi_{11}+\pi_{10}\right)\right\}$. Suppose $m \pi_{c}$ markers are genotyped in stage II children. As mentioned in the text, we use slightly different test statistics for analyzing stage I and stage II offspring data. For stage I, all available maternal and offspring genotype data is utilized. The likelihood function, denoted as $L^{I}\left(\beta_{1}, p_{a} ; G^{c}, G^{m}\right)$, is provided in Section 2.2. Denote the corresponding score statistic as $S_{I}$. For stage II, we assume that only offspring genotype is available. Thus, the score statistic using stage-II data only is based on a slightly different likelihood:

$$
\begin{aligned}
L^{I I}\left(\beta_{1}, p_{a} ; G^{c}\right)= & \prod_{i=1}^{n_{21}} \frac{e^{\beta_{1} G_{i}^{c}} p\left(G_{i}^{c} \mid Y_{i}=0\right)}{\sum_{G^{c}} e^{\beta_{1} G^{c}} p\left(G^{c} \mid Y=0\right)} \\
& \times \prod_{j=1}^{n_{20}} p\left(G_{j}^{c} \mid Y_{j}=0\right),
\end{aligned}
$$

where $n_{20}=n_{21}=n\left\{1-\pi_{1}\left(\pi_{11}+\pi_{10}\right) / 2\right\}$ is the number stage-II case or control children. Denote the resultant statistic as $S_{I I}$. For the joint analysis of stage I and stage II data in the sense of (Skol et al., 2006), we maximize the joint likelihood function $L^{J}\left(\beta_{1}, p_{a}\right)=L^{I}\left(\beta_{1}, p_{a} ; G^{c}, G^{m}\right) L^{I I}\left(\beta_{1}, p_{a} ; G^{c}\right)$. Let 
$l_{\beta_{1}}^{J}\left(\hat{p}_{a}\right)=\partial \log L^{J}\left(\beta_{1}=0, \hat{p}_{a}\right) / \partial \beta_{1}$. The test of hypothesis $\beta_{1}=0$ is based on the statistic $S_{J}=$ $l_{\beta_{1}}^{J}\left(\hat{p}_{a}\right) / \operatorname{var}^{1 / 2}\left\{l_{\beta_{1}}^{J}\left(\hat{p}_{a}\right)\right\}$, which is a standard normal random variable. We calculate the power as $p_{h_{a}}\left(\left|S_{I}\right| \geq c_{I},\left|S_{J}\right| \geq\right.$ $\left.c_{J}\right)$. The critical points $c_{I}$ and $c_{J}$ are determined based on $p_{h_{0}}\left(\left|S_{I}\right| \geq c_{I}\right)=\pi_{m}$ and $p_{h_{0}}\left(\left|S_{1}\right| \geq c_{1},\left|S_{J}\right| \geq c_{J}\right)=\alpha$. For testing $500 \mathrm{k}$ SNPs, $\alpha$ could be $10^{-7}$. The subscripts $h_{a}$ and $h_{0}$ denote that the calculation is under the alternative and null hypotheses, respectively.

Below we describe how to calculate the joint distribution of $\left(S_{I}, S_{J}\right)$, which is used for calculating the power $p_{h_{a}}\left(\left|S_{I}\right| \geq c_{I},\left|S_{J}\right| \geq c_{J}\right)$. First, we obtain score functions for $\beta_{1}, l_{\beta_{1}}^{I}\left(\beta_{1}=0, \hat{p}_{a}\right)$ and $l_{\beta_{1}}^{I I}\left(\beta_{1}=0, \hat{p}_{a}\right)$, and those for $p_{a}$, $l_{p_{a}}^{I}\left(\beta_{1}=0, \hat{p}_{a}\right)$ and $l_{p_{a}}^{I I}\left(\beta_{1}=0, \hat{p}_{a}\right)$, separately for stage I and stage II data. Below, $n_{21}$ and $n_{20}$ are the respective numbers of stage II cases and controls, $n_{1}^{c}$ and $n_{0}^{c}$ are the respective numbers of complete stage I case and control mother-child pairs, and $n_{1}$ and $n_{0}$ are the total respective numbers of stage I cases and controls. We omit " $\beta_{1}=0$ " in all score functions.

$$
\begin{aligned}
l_{\beta_{1}}^{I}\left(p_{a}\right)= & \sum_{i=1}^{n_{1}^{c}}\left\{G_{i}^{c}-\mathrm{E}\left(G^{c}\right)\right\}+\sum_{i=n_{1}^{c}+1}^{n_{1}}\left\{\mathrm{E}\left(G^{c} \mid G_{i}^{m}\right)-\mathrm{E}\left(G^{c}\right)\right\} \\
l_{\beta_{1}}^{I I}\left(p_{a}\right)= & \sum_{i=1}^{n_{21}}\left\{G_{i}^{c}-\mathrm{E}\left(G^{c}\right)\right\} \\
l_{\mathrm{p}_{a}}^{I}\left(p_{a}\right)= & \sum_{i=1}^{n_{1}^{c}}\left[\frac{\partial \log \mathrm{p}\left(G_{i}^{c}, G_{i}^{m}\right)}{\partial p_{a}}-\mathrm{E}\left\{\frac{\partial \log p\left(G^{c}\right)}{\partial p_{a}}\right\}\right] \\
& +\sum_{i=n_{1}^{c}+1}^{n_{1}}\left[\mathrm{E}\left\{\frac{\partial \log p\left(G^{c}, G_{i}^{m}\right)}{\partial p_{a}} \mid G_{i}^{m}\right\}\right. \\
& \left.\left.-\mathrm{E}\left\{\frac{\partial \log p\left(G^{c}\right)}{\partial p_{a}}\right\}\right]\right] \\
& +\sum_{j=1}^{n_{0}^{c}} \frac{\partial \log \mathrm{p}\left(G_{j}^{c}, G_{j}^{m}\right)}{\partial p_{a}}+\sum_{j=n_{0}^{c}+1}^{n_{0}} \frac{\partial \log \mathrm{p}\left(G_{j}^{m}\right)}{\partial p_{a}} \\
& +\sum_{j=1}^{n_{21}}\left[\frac{\partial \log p\left(G_{i}^{c}\right)}{\partial p_{a}}-\mathrm{E}\left\{\frac{\partial \log p\left(G^{c}\right)}{\partial p_{a}}\right\}\right] \\
& \left.\sum_{i=1}^{n_{20}}\right\} \\
l_{\mathrm{p}_{a}}^{I I}\left(p_{a}\right) & \frac{\partial p_{a}}{\log p\left(G_{j}^{c}\right)}
\end{aligned}
$$

Our test statistics for the stage I and joint analyses are constructed based on $l_{\beta_{1}}^{I}\left(\hat{p}_{a}^{I}\right)$ and $l_{\beta_{1}}^{J}\left(\hat{p}_{a}\right)=l_{\beta_{1}}^{I}\left(\hat{p}_{a}^{J}\right)+l_{\beta_{1}}^{I I}\left(\hat{p}_{a}^{J}\right)$, where $\hat{p}_{a}^{I}$ and $\hat{p}_{a}^{J}$ are solutions to equations $l_{\mathrm{p}_{a}}^{I}\left(p_{a}\right)=0$ and $l_{\mathrm{p}_{a}}^{J}\left(p_{a}\right)=0$, respectively. To obtain their asymptotic variance, we perform the following Taylor series expansion:

$$
l_{\beta_{1}}^{I}\left(\hat{p}_{a}^{I}\right) \approx l_{\beta_{1}}^{I}\left(p_{a}\right)-\frac{\partial l_{\beta_{1}}^{I}\left(p_{a}\right)}{\partial p_{a}}\left\{\frac{\partial l_{\mathrm{p}_{a}}^{I}\left(p_{a}\right)}{\partial p_{a}}\right\}^{-1} l_{\mathrm{p}_{a}}^{I}\left(p_{a}\right),
$$

$$
l_{\beta_{1}}^{J}\left(\hat{p}_{a}^{J}\right) \approx l_{\beta_{1}}^{J}\left(p_{a}\right)-\frac{\partial l_{\beta_{1}}^{J}\left(p_{a}\right)}{\partial p_{a}}\left\{\frac{\partial l_{\mathrm{p}_{a}}^{J}\left(p_{a}\right)}{\partial p_{a}}\right\}^{-1} l_{\mathrm{p}_{a}}^{J}\left(p_{a}\right) .
$$

Recognizing that $-\partial l_{\mathrm{p}_{a}}^{I}\left(p_{a}\right) / \partial p_{a}=\operatorname{var}\left\{l_{\mathrm{p}_{a}}^{I}\left(p_{a}\right)\right\}$, $\partial l_{\beta_{1}}^{I}\left(p_{a}\right) / \partial p_{a}=\operatorname{cov}\left\{l_{\beta_{1}}^{I}\left(p_{a}\right), l_{\mathrm{p}_{a}}^{I}\left(p_{a}\right)\right\}$, and similar results for $l_{\mathrm{p}_{a}}^{J}$, we can obtain variances for $l_{\beta_{1}}^{J}\left(\hat{p}_{a}^{J}\right)$ and $l_{\beta_{1}}^{I}\left(\hat{p}_{a}^{I}\right)$ (see below for details). Based on these Taylor expansion results, we can obtain the covariance of the two score functions. Thus, we obtain the joint distribution of the two test statistics $S_{I}$ and $S_{J}$, which follows a bivariate normal distribution. The power can then be calculated.

Let $l=0,1$ denote case-control status and $k=I, I I$ denote stages I and II. Define $n_{i j}^{k l c}=\sum_{s=1}^{n_{l}} I_{g_{s}^{m}=i, g_{s}^{c}=j}$, $n_{j}^{k l c-}=\sum_{s=1}^{n_{l}} I_{g_{s}^{m}=j}$, and $n_{j}^{k l}=\sum_{s=1}^{n_{l}} I_{g_{s}^{c}=j}, i, j=0,1,2$. Under the null hypothesis, $p_{a}$ can be estimated as

$$
\hat{p}_{a}^{I}=\frac{n_{I}^{*}}{n_{I}^{\#}} \quad \text { and } \quad \hat{p}_{a}^{J}=\frac{n_{I}^{*}+n_{I I}^{*}}{n_{I}^{\#}+n_{I I}^{\#}}
$$

where

$$
\begin{aligned}
n_{I}^{*}= & n_{01}^{11 c}+n_{10}^{11 c}+n_{11}^{11 c}+2\left(n_{12}^{11 c}+n_{21}^{11 c}\right)+3 n_{22}^{11 c} \\
& +n_{1}^{11 c-}+2 n_{2}^{11 c-}+n_{01}^{10 c}+n_{10}^{10 c}+n_{11}^{10 c} \\
& +2\left(n_{12}^{10 c}+n_{21}^{10 c}\right)+3 n_{22}^{10 c}+n_{1}^{10 c-}+2 n_{2}^{10 c-} \\
n_{I}^{\#}= & 3\left(n_{00}^{11 c}+n_{01}^{11 c}+n_{10}^{11 c}\right)+2 n_{11}^{11 c}+3\left(n_{12}^{11 c}\right. \\
& \left.+n_{21}^{11 c}+n_{22}^{11 c}\right)+2\left(n_{0}^{11 c-}+n_{1}^{11 c-}+n_{2}^{11 c-}\right) \\
& +3\left(n_{00}^{10 c}+n_{01}^{10 c}+n_{10}^{10 c}\right)+2 n_{11}^{10 c}+3\left(n_{12}^{10 c}\right. \\
& \left.+n_{21}^{10 c}+n_{22}^{10 c}\right)+2\left(n_{0}^{10 c-}+n_{1}^{10 c-}+n_{2}^{10 c-}\right), \\
n_{I I}^{*}= & n_{1}^{21}+2 n_{2}^{21}+n_{1}^{20}+2 n_{2}^{20} \\
n_{I I}^{\#}= & 2\left(n_{0}^{21}+n_{1}^{21}+n_{2}^{21}\right)+2\left(n_{0}^{20}+n_{1}^{20}+n_{2}^{20}\right) .
\end{aligned}
$$

We verified that the two estimators are consistent under the null hypothesis.

Let $c_{1}^{I}=\lim _{n_{1} \rightarrow \infty} n_{1}^{-1} \partial l_{p_{a}}^{I} / \partial p_{a}, \quad c_{1}^{J}=$ $\lim _{n \rightarrow \infty} n^{-1} \partial l_{p_{a}}^{J} / \partial p_{a}, \quad c_{2}^{I}=\lim _{n_{1} \rightarrow \infty} n_{1}^{-1} \partial l_{\beta_{1}}^{I} / \partial p_{a}$, and $c_{1}^{J}=\lim _{n \rightarrow \infty} n^{-1} \partial l_{\beta_{1}}^{J} / \partial p_{a}$. With the above estimated MAF $\hat{p}_{a}^{I}$ and $\hat{p}_{a}^{J}$, we obtained the correlation between $l_{\beta_{1}}^{I}\left(\hat{p}_{a}^{I}\right)$ and $l_{\beta_{1}}^{J}\left(\hat{p}_{a}^{J}\right), \operatorname{Cor}\left\{l_{\beta_{1}}^{I}\left(\hat{p}_{a}^{I}\right), l_{\beta_{1}}^{J}\left(\hat{p}_{a}^{J}\right)\right\}$, as follows under the null:

$$
\sqrt{\pi_{1}} \sqrt{\frac{\lim _{n_{1} \rightarrow \infty} \frac{1}{n_{1}} \operatorname{Var}\left(l_{\beta_{1}}^{I}\left(p_{a}\right)\right)+\frac{\left(c_{2}^{I}\right)^{2}}{c_{1}^{I}}}{\lim _{n_{1} \rightarrow \infty} \frac{1}{n} \operatorname{Var}\left(l_{\beta_{1}}^{J}\left(p_{a}\right)\right)+\frac{\left(c_{2}^{J}\right)^{2}}{c_{1}^{J}}}}
$$

which can be further simplified as

$$
\sqrt{\pi_{1}} \sqrt{\frac{\frac{1}{2}\left(1+3 \pi_{11}\right) p_{a}\left(1-p_{a}\right)+\frac{\left(1+\pi_{11}\right)^{2}}{\left(2-\frac{3 \pi_{1}\left(1-\pi_{11}\right)}{2}\right) p_{a}\left(1-p_{a}\right)+\frac{\left(2-\pi_{1}\left(1-\pi_{11}\right)\right)^{2}}{p_{a}\left(1-\pi_{10}\right)}}}{\pi_{1}\left(\pi_{11}+\pi_{10}\right)-\frac{4+\pi_{1}\left(\pi_{11}+\pi_{10}\right)}{p_{a}\left(1-p_{a}\right)}}} .
$$

Under the alternative hypothesis, we can not obtain a similarly simple formula. Furthermore, under the alternative, $\hat{p}_{a}^{I}$ 
and $\hat{p}_{a}^{J}$ above are no longer unbiased estimators of $p_{a}$. We thus calculated $\operatorname{Cor}\left\{l_{\beta_{1}}^{I}\left(\hat{p}_{a}^{I}\right), l_{\beta_{1}}^{J}\left(\hat{p}_{a}^{J}\right)\right\}$ as

$$
\frac{\operatorname{Cov}\left\{l_{\beta_{1}}^{I}\left(\hat{p}_{a}^{I}\right), l_{\beta_{1}}^{J}\left(\hat{p}_{a}^{J}\right)\right\}}{\sqrt{\operatorname{Var}\left\{l_{\beta_{1}}^{I}\left(\hat{p}_{a}^{I}\right)\right\} \operatorname{Var}\left\{l_{\beta_{1}}^{J}\left(\hat{p}_{a}^{J}\right)\right\}}} .
$$

Let $p^{1}\left(G^{m}, G^{c}\right)=p\left(G^{m}, G^{c} \mid Y=1\right), p^{0}\left(G^{m}, G^{c}\right)=$ $p\left(G^{m}, G^{c} \mid Y=0\right), p^{1}\left(G^{m}\right)=p\left(G^{m} \mid Y=1\right), p^{0}\left(G^{m}\right)=$ $p\left(G^{m} \mid Y=0\right), p^{1}\left(G^{c}\right)=p\left(G^{c} \mid Y=1\right), p^{0}\left(G^{c}\right)=p\left(G^{c} \mid Y=0\right)$. These probabilities represent the proportion of genotypes in the real data. Then, the covariance $\operatorname{Cov}\left\{l_{\beta_{1}}^{I}\left(\hat{p}_{a}^{I}\right), l_{\beta_{1}}^{J}\left(\hat{p}_{a}^{J}\right)\right\}$ can be calculated as

$$
\begin{aligned}
\operatorname{Cov} & \left\{l_{\beta_{1}}^{J}\left(p_{a}^{J}\right)-\frac{c_{2}^{J}}{c_{1}^{J}} l_{\mathrm{p}_{a}}^{J}\left(p_{a}^{J}\right), l_{\beta_{1}}^{I}\left(p_{a}^{I}\right)-\frac{c_{2}^{I}}{c_{1}^{I}} l_{\mathrm{p}_{a}}^{I}\left(p_{a}^{I}\right)\right\} \\
= & \operatorname{Cov}\left\{l_{\beta_{1}}^{I}\left(p_{a}^{I}\right), l_{\beta_{1}}^{J}\left(p_{a}^{J}\right)\right\}-\frac{c_{2}^{J}}{c_{1}^{J}} \operatorname{Cov}\left\{l_{\mathrm{p}_{a}}^{J}\left(p_{a}^{J}\right), l_{\beta_{1}}^{I}\left(p_{a}^{I}\right)\right\} \\
& -\frac{c_{2}^{I}}{c_{1}^{I}} \operatorname{Cov}\left\{l_{\mathrm{p}_{a}}^{I}\left(p_{a}^{I}\right), l_{\beta_{1}}^{J}\left(p_{a}^{J}\right)\right\} \\
& +\frac{c_{2}^{I}}{c_{1}^{I}} \frac{c_{2}^{J}}{c_{1}^{J}} \operatorname{Cov}\left\{l_{\mathrm{p}_{a}}^{J}\left(p_{a}^{J}\right), \quad l_{p_{a}}^{I}\left(p_{a}^{I}\right)\right\},
\end{aligned}
$$

where $\left(n \pi_{1}\right)^{-1} \operatorname{Cov}\left\{l_{\beta_{1}}^{I}\left(p_{a}^{I}\right), l_{\beta_{1}}^{J}\left(p_{a}^{J}\right)\right\}$ can be calculated as

$$
\begin{aligned}
& \sum_{G^{c}} G_{c}^{2} p^{1}\left(G^{c}\right)-\left\{\sum_{G^{c}} G_{c} p^{1}\left(G^{c}\right)\right\}^{2} \\
& +\sum_{G^{m}} \mathrm{E}_{p_{a}^{I}}\left(G^{c} \mid G^{m}\right) \mathrm{E}_{p_{a}^{J}}\left(G^{c} \mid G^{m}\right) p^{1}\left(G^{m}\right) \\
& \quad-\left\{\sum_{G^{m}} \mathrm{E}_{p_{a}^{I}}\left(G^{c} \mid G^{m}\right) p^{1}\left(G^{m}\right)\right\}\left\{\sum_{G^{m}} \mathrm{E}_{p_{a}^{J}}\left(G^{c} \mid G^{m}\right) p^{1}\left(G^{m}\right)\right\} .
\end{aligned}
$$

Note that $E\left(G^{c} \mid G^{m}\right)$ is a function of $p_{a}$. Furthermore, $\left(n \pi_{1}\right)^{-1} \operatorname{Cov}\left\{l_{\mathrm{p}_{a}}^{J}\left(p_{a}^{J}\right), l_{\beta_{1}}^{I}\left(p_{a}^{I}\right)\right\}$ can be obtained as

$$
\begin{aligned}
& \sum_{G^{c}, G^{m}} G_{c} \frac{\log \mathrm{p}_{p_{a}^{J}}\left(G^{c}, G^{m}\right)}{\partial p_{a}} p^{1}\left(G^{c}, G^{m}\right) \\
& \quad-\left\{\sum_{G^{c}, G^{m}} \frac{\log \mathrm{p}_{p_{a}^{J}}\left(G^{c}, G^{m}\right)}{\partial p_{a}} p^{1}\left(G^{c}, G^{m}\right)\right\}\left\{\sum_{G^{c}}\left(G_{c} p^{1}\left(G^{c}\right)\right)\right\} \\
& +\sum_{G^{m}} \frac{\log \sum_{G^{c}} \mathrm{p}_{p_{a}^{J}}\left(G^{c}, G^{m}\right)}{\partial p_{a}} \mathrm{E}_{p_{a}^{I}}\left(G^{c} \mid G^{m}\right) p^{1}\left(G^{m}\right) \\
& -\left\{\sum_{G^{m}} \mathrm{E}_{p_{a}^{J}} \frac{\log \sum_{g^{c}} \mathrm{p}\left(G^{c}, G^{m}\right)}{\partial p_{a}} p^{1}\left(G^{m}\right)\right\} \\
& \quad \times\left\{\sum_{G^{m}} \mathrm{E}_{p_{a}^{I}}\left(G^{c} \mid G^{m}\right) p^{1}\left(G^{m}\right)\right\} .
\end{aligned}
$$

Similarly, we calculate $\left(n \pi_{1}\right)^{-1} \operatorname{Cov}\left\{l_{\mathrm{p}_{a}}^{I}\left(p_{a}^{I}\right), l_{\beta_{1}}^{J}\left(p_{a}^{J}\right)\right\}$ as

$$
\sum_{G^{c}, G^{m}} G_{c} \frac{\log p_{p_{a}^{I}}\left(G^{c}, G^{m}\right)}{\partial p_{a}} p^{1}\left(G^{c}, G^{m}\right)
$$

$$
\begin{aligned}
& -\left\{\sum_{G^{c}, G^{m}} \frac{\log \mathrm{p}_{p_{a}^{I}}\left(G^{c}, G^{m}\right)}{\partial p_{a}} p^{1}\left(G^{c}, G^{m}\right)\right\} \\
& \times\left\{\sum_{G^{c}} G_{c} p^{1}\left(G^{c}\right)\right\} \\
& +\sum_{G^{m}} \frac{\log \sum_{G^{c}} \mathrm{p}_{p_{a}^{I}}\left(G^{c}, G^{m}\right)}{\partial p_{a}} \mathrm{E}_{p_{a}^{J}}\left(G^{c} \mid G^{m}\right) p^{1}\left(G^{m}\right) \\
& -\left\{\sum_{G^{m}}\left(\mathrm{E}_{p_{a}^{I}} \frac{\log \sum_{G^{c}} \mathrm{p}\left(G^{c}, G^{m}\right)}{\partial p_{a}} p^{1}\left(G^{m}\right)\right)\right\} \\
& \times\left\{\sum_{G^{m}}\left(\mathrm{E}_{p_{a}^{I}}\left(G^{c} \mid G^{m}\right) p^{1}\left(G^{m}\right)\right)\right\} .
\end{aligned}
$$

Lastly, we calculate $\left(n \pi_{1}\right)^{-1} \operatorname{Cov}\left\{l_{\mathrm{p}_{a}}^{I}\left(p_{a}^{I}\right), l_{\mathrm{p}_{a}}^{J}\left(p_{a}^{J}\right)\right\}$ as

$$
\begin{aligned}
& \sum_{G^{c}, G^{m}} \frac{\log \mathrm{p}_{p_{a}^{J}}\left(G^{c}, G^{m}\right)}{\partial p_{a}} \frac{\log \mathrm{p}_{p_{a}^{I}}\left(G^{c}, G^{m}\right)}{\partial p_{a}} p^{1}\left(G^{c}, G^{m}\right) \\
& -\left\{\sum_{G^{c}, G^{m}} \frac{\log \mathrm{p}_{p_{a}^{I}}\left(G^{c}, G^{m}\right)}{\partial p_{a}} p^{1}\left(G^{c}, G^{m}\right)\right\} \\
& \quad \times\left\{\sum_{G^{c}} \frac{\log \mathrm{p}_{p_{a}^{J}}\left(G^{c}, G^{m}\right)}{\partial p_{a}} p^{1}\left(G^{c}, G^{m}\right)\right\} \\
& +\sum_{G^{m}} \frac{\log \sum_{G^{c}} \mathrm{p}_{p_{a}^{I}}\left(G^{c}, G^{m}\right)}{\partial p_{a}} \frac{\log \sum_{G^{c}} \mathrm{p}_{p_{a}^{J}}\left(G^{c}, G^{m}\right)}{\partial p_{a}} p^{1}\left(G^{m}\right) \\
& -\left\{\sum_{G^{m}} \frac{\log \sum_{G^{c}} \mathrm{p}\left(G^{c}, G^{m}\right)}{\partial p_{a}} p^{1}\left(G^{m}\right)\right\} \\
& \times\left\{\sum_{G^{m}} \frac{\log \sum_{G^{c}} \mathrm{p}_{p_{a}^{J}}\left(G^{c}, G^{m}\right)}{\partial p_{a}} p^{1}\left(G^{m}\right)\right\} .
\end{aligned}
$$

For the score test of the maternal effect with genotype data available for all cases and controls, we obtain $\operatorname{Cor}\left\{l_{\beta_{1}}^{I}\left(\hat{p}_{a}^{I}\right), l_{\beta_{1}}^{J}\left(\hat{p}_{a}^{J}\right)\right\}=\sqrt{\pi_{1}}$ under the null hypothesis. Note that $p_{a}$ can be estimated as

$$
\hat{p}_{a}=\frac{1}{4 n}\left[\sum_{i=1}^{n_{1}}\left(I_{G_{i}^{m}=1}+2 I_{G_{i}^{m}=2}\right) \sum_{i=1}^{n_{0}}\left(I_{G_{i}^{m}=1}+2 I_{G_{i}^{m}=2}\right)\right] .
$$

The score statistic also takes a very simple form:

$$
\begin{aligned}
l_{\beta_{1}}\left(\beta_{1}=0, p_{a}\right)= & \sum_{i=1}^{n_{1}} G_{i}^{m}-n_{1} 2 \hat{p}_{a} \\
= & \sum_{i=1}^{n_{1}} G_{i}^{m}-\frac{1}{2}\left[\sum _ { i = 1 } ^ { n _ { 1 } } \left(I_{G_{i}^{m}=1}\right.\right. \\
& \left.\left.+2 I_{G_{i}^{m}=2}\right)+\sum_{i=1}^{n_{0}}\left(I_{G_{i}^{m}=1}+2 I_{G_{i}^{m}=2}\right)\right]
\end{aligned}
$$

Under the alternative hypothesis, the variance of the score function $l_{\beta_{1}}\left(\beta_{1}=0, p_{a}\right), n_{1}^{-1} \operatorname{Var}\left\{l_{\beta_{1}}\left(\beta_{1}=0, p_{a}\right)\right\}$, can also 
be greatly simplified:

$$
\begin{aligned}
& \frac{1}{4} p_{1}^{1}\left(1-p_{1}^{1}\right)+p_{2}^{1}\left(1-p_{2}^{1}\right)+\frac{1}{4} p_{1}^{0}\left(1-p_{1}^{0}\right)+p_{2}^{0}\left(1-p_{2}^{0}\right) \\
& \quad-p_{1}^{1} p_{2}^{1}-p_{1}^{0} p_{2}^{0}
\end{aligned}
$$

where $p_{i}^{k}=p\left(G^{m}=i \mid Y=k\right)$ with $k=0,1$ indicating case-control status and $i=0,1,2$ being the genotype score.

\section{ACKNOWLEDGEMENTS}

Drs. Jinbo Chen and Haitao Zheng were supported by NIH grant ES016626. Dr. Cuilin Zhang was supported by the Intramural Research Program of the Eunice Kennedy Shriver National Institute of Child Health \& Human Development, National Institutes of Health.

\section{Received 14 January 2010}

\section{REFERENCES}

Armitage, P. (1955). Tests for Linear Trends in Proportions and Frequencies. Biometrics 11:375-386.

Chen, J. and Chatterjee, N. (2007). Exploiting Hardy-Weinberg equilibrium for efficient screening of single SNP associations from case-control studies. Human Heredity 63:196-204.

Chen, J., Zheng, H., and Wilson, M. L. (2009). Likelihood ratio tests for maternal and fetal genetic effects on obstetric complications. Genetic Epidemiology 33:526-538.

Chen J., Zheng, H., Wilson, M. L., and Kraft, P. (2009). Testing Hardy-Weinberg equilibrium using mother-child case-control samples. Genetic Epidemiology 33:539-548.

Cohen, I. L., Liu, X., Lewis, M. E. S., Chudley, A., ForsterGibson, C., Gonzalez, M., Jenkins, E. C., Brown, W. T., and Holden, J. J. A. (2010). Autism severity is associated with child and maternal MAOA genotypes. Clinical Genecis dol:10.111/j.13990004.2010.01471.x.

Goddard, K. A., Tromp, G., Romero, R., Olson, J. M., Lu, Q., Xu, Z., Parimi, N., Nien, J. K., Gomez, R., Behnke, E., Solari, M., Espinoza, J., Santolaya, J., Chaimorapongsa, T., Lenk, G. M., Volkenant, K., Anant, M. K., Salisbury, B. A., Carr, J., Lee, M. S., Vovis, G. F., and Kuivaniemi, H. (2007). Candidate-gene association study of mothers with preeclampsia, and their infants, analyzing 775 SNPs in 190 genes. Human Heredity 63:1-16.

James, S. J., Melnyk, S., Jernigan, S., Pavliv, O., Trusty, T., Lehman, S., Seidel, L., Gaylor, D. W., and Cleves, M. A. (2010). A functional polymorphism in the reduced folate carrier gene and DNA hypomethylation in mothers of children with autism. American Journal of Medical Genetics 153B:1209-1220.

Kanayama, N., Takahashi, K., Matsuura, T., Sugimura, M., Kobayashi, T., Moniwa, N., Tomita, M., and Nakayama, K. (2003). Deficiency in p57Kip2 expression induces preeclampsia-like symptoms in mice. Molecular Human Reproduction 8:1129-1135.

Petry, C. J., Ong, K. K., and Dunger, D. B. (2007). Does the fetal genotype affect maternal physiology during pregnancy? Trends in Molecular Medicine 13:414-421.

Petry, C. J., Evans, M. L., Wingate, D. L., Ong, K. K., Reik, W., Constancia, M., and Dunger, D. B. (2010). Raised late pregnancy glucose concentrations in mice carrying pups with targeted disruption of H19. Diabetes 59:282-286.
Romero, R., Velez, Edwards D. R., Kusanovic, J. P., Hassan, S. S., Mazaki-Tovi, S., Vaisbuch, E., Kim, C. J., Chaiworapongsa, T., Pearce, B. D., Friel, L. A., Bartlett, J., Anant, M. K., Salisbury, B. A., Vovis, G. F., Lee, M. S., Gomez, R., Behnke, E., Oyarzun, E., Tromp, G., Williams, S. M., and Menon, R. (2010). Identification of fetal and maternal single nucleotide polymorphisms in candidate genes that predispose to spontaneous preterm labor with intact membranes. American Journal of Obstetric Gynecology 202:431.e1-431.e34.

Saftlas, A. F., Beydoun, H., and Triche, E. (2005). Immunogenetic determinants of preeclampsia and related pregnancy disorders: a systematic review. Obstetrical Gynecology 106:162-172.

Satagopan, J. M., Venkatraman, E. S., and Begg, C. B. (2004). Two-stage designs for gene-disease association studies with sample size constraints. Biometrics 60:589-597. MR2089433

Skol, A. D., Scott, L. J., Abechisis, G. R., and Bonhnke, M. (2007). Optimal designs for two-stage genome-wide association studies. Genetic Epidemiology 31:776-788.

Steffen, K. M., Cooper, M. E., Shi, M., Caprau, D., Simhan, H. N., Dagle, J. M., Marazita, M. L., and Murray, J. C. (2007). Maternal and fetal variation in genes of cholesterol metabolism is associated with preterm delivery. Journal of Perinatology 27:672-680.

Takimoto, E., Ishida, J., Sugiyama, F., Horiguchi, H., Murakami, K., and FukAmizu, A. (1996). Hypertension induced in pregnant mice by placental renin and maternal angiotensinogen. Science 274:995-998.

Wang, H. S., Thomas, D. C., and Stram, D. O. (2006). Optimal twostage genotyping designs for genome-wide association scans. Genetic Epidemiology 30:356-368.

Wangler, M. F., Chang, A. S., Moley, K. H., Feinberg, A. P., and DeBAun, M. R. (2005). Factors associated with preterm delivery in mothers of children with Beckwith-Wiedemann syndrome: a case cohort study from the BWS registry. American Journal of Medical Genetics 134:187-191.

WeinberG, C. R. and Shi, M. (2009). The genetics of preterm birth: using what we know to design better association studies. American Journal of Epidemiology 170:1373-1381.

Weinberg, C. R., Wilcox, A. J., and Lie, R. T. (1998). A log-linear approach to case-parent triad data: Assessing effects of disease genes that act directly or through maternal effects, and may be subject to parental imprinting. American Journal of Human Genetics 62:969 978

Wilcox, A. J., Weinberg, C. R., and Lie, R. T. (1998). Distinguishing the effects of maternal and offspring genes through studies of case-parent triads. American Journal of Epidemiology 148:893-901.

ZAykin, D. and Zhivotovsky, L. A. (2005). Ranks of genuine associations in whole-genome scans. Genetics 17:813-283.

Haitao Zheng

Department of Biostatistics and Epidemiology

University of Pennsylvania School of Medicine

Philadelphia, PA 19104

USA

E-mail address: htzheng@gmail.com

Cuilin Zhang

Epidemiology Branch, Division of Epidemiology

Statistics, and Prevention Research

National Institute of Child Health and Human Development 6100 Executive Blvd, Room 7B03

MSC 7510, 9000

USA

E-mail address: zhangcu@mail.nih.gov 


\section{Yun Lu}

Department of Biostatistics and Epidemiology University of Pennsylvania School of Medicine Philadelphia, PA 19104

USA

E-mail address: luyun@mail.med.upenn.edu
Jinbo Chen

Department of Biostatistics and Epidemiology

University of Pennsylvania School of Medicine

Philadelphia, PA 19104

USA

E-mail address: jinboche@mail.med.upenn.edu 